\title{
Periodontal disease and pregnancy outcome, are we still in the same place?
}

\begin{abstract}
There is a general concern about the relationship between periodontal disease and pregnancy, although the lack of evidence in the studies is significant, there is a population that must receive attention. It is important that dentists and gyneco-obstetrics work together to keep oral health in pregnant women and also improve their oral hygiene. This minireview highlights the importance of periodontal disease, pathogens involved, treatment and dental management in pregnant women.
\end{abstract}

Keywords: periodontal disease, pregnancy, oral health
Volume 8 Issue 2 - 2017

\author{
Maria Gabriela Acosta de Camargo,' Maria \\ Isabel Acosta ${ }^{2,3}$ \\ 'Department of Pediatric Dentistry, University of Carabobo, \\ Venezuela \\ ${ }^{2}$ Human Reproduction Medicine, King Juan Carlos University, \\ Spain \\ ${ }^{3}$ Department of Obstetrics and Gynecology, University \\ Centrooccidental Lisandro Alvarado, Venezuela
}

Correspondence: Acosta de Camargo Maria Gabriela Department of Pediatric Dentistry, University of Carabobo, Venezuela, Email gabrieladecamargo@yahoo.com

Received: July 26, 2017 | Published: September 22, 2017

\section{Discussion}

Dental health can be the reflection of the general health in a patient, considering the incidence of oral manifestations on a big group of diseases and also the consequences that an oral pathology can have in the general health. The relationship of pathogens in the oral cavity and a great number of diseases is well described; being the most known endocarditis. However many other relationships have been described. ${ }^{1}$ Among the pathologies in oral cavity one of special interest during pregnancy is periodontal disease (PD). This is a multifactorial inflammatory condition in which inappropriate interaction between the host immune response and specific groups of bacterial pathogens leads to destruction of connective and bone tissues supporting the tooth. Dissemination of pathogens, toxins, and immune complexes from and to periodontal lesions is at the basis of the increasingly recognized association between PD and various systemic diseases. ${ }^{1}$ PD is likely to cause $19 \%$ increase in the risk of cardiovascular disease, and this increase in relative risk reaches $44 \%$ among individuals aged 65 years and over. Type 2 diabetic individuals with severe form of periodontal disease have 3.2 times greater mortality risk compared with individuals with no or mild periodontitis. Periodontal therapy has been shown to improve glycemic control in type 2 diabetic subjects. ${ }^{2}$ Also it has been known for some time that relationships exist between periodontal disease and other medical conditions such as kidney disease, and rheumatoid arthritis. ${ }^{3}$ The typical clinical signs in chronic periodontitis occur mainly as a result of activation between host-derived immune and inflammatory defense mechanisms. IL-1 and TNF induce expression of other mediators that amplify the inflammatory response, such as prostaglandins, and lead to production of lytic enzymes and stimulate the production of chemokines. ${ }^{4}$ Furthermore, periodontitis is related to maternal infection, preterm birth, low birth weight, and preeclampsia. ${ }^{2}$ In recent years, research linking periodontitis to the risk of adverse birth outcomes has resulted in an increased interest in the topic of oral health during pregnancy.
Preterm birth (PB) is a primary public health challenge in both developed and underdeveloped nations. ${ }^{5,6}$ In 2016 Mattheus et al. recommended promoting legislation to include dental coverage for pregnant women regardless of their current medical insurance. This has the potential to increase the utilization of dental services by women for both preventive and restorative care, ${ }^{7}$ showing the possibility of a relationship among the lack of access to dental coverage and the complications during pregnancy in Hawaii. Other authors have also shown that the incidence of pre-term low birth weight still prevails in developed as well as developing countries though the numbers may vary. ${ }^{8}$ It is also worth mentioning that some studies have shown a stronger relationship between periodontitis and perinatal complication among vulnerable populations like refugees. ${ }^{9}$ Pregnancy sometimes has adverse outcomes including low birthweight $(<2500 \mathrm{~g})$, pre-term birth ( $<37$ weeks), growth restriction, pre-eclampsia, miscarriage and/ or stillbirth. Maternal periodontitis directly and/or indirectly has the potential to influence the health of the foetal-maternal unit. ${ }^{10}$ Preterm birth accounts for $70 \%$ of perinatal mortality, nearly $50 \%$ of long term neurological morbidity, and a significant impact on health care costs. There is evidence that spontaneous preterm labor and preterm birth are associated with intrauterine infection due to abnormal genital and/ or oral colonization. ${ }^{11}$ Preterm birth and small for gestational age are strong indicators of neonatal adverse outcomes ${ }^{12}$ and is a significant causative factor of infant and child morbidity and mortality. Preterm birth complications are estimated to be the second most common cause of death in children less than 5 years old. ${ }^{13}$ It is estimated that six million perinatal deaths occur every year worldwide, with premature birth being the main cause. ${ }^{14}$

Despite improvements in obstetric care, rates of preterm birth have not decreased during the last 10 years. The etiological role of maternal infection, either in the genital tract or elsewhere, on preterm delivery remains unclear. PD is one of the most common chronic infectious diseases. This type of infection is caused primarily by Gram-negative 
anaerobic, and microaerophilic bacteria that colonize the subgingival area and produce significant amounts of proinflammatory cytokines, mainly interleukin 1 beta and interleukin 6, prostaglandin E2, and tumor necrosis factor alpha. PD may therefore influence PB through an indirect mechanism involving inflammatory mediators or through a direct bacterial assault on the amnion. ${ }^{5}$ Two pathogenic mechanisms have been proposed to explain this association. The direct pathway is based on the presence of gram-negative anaerobic bacteremia originating in the gingival biofilm, whereas the indirect pathway involves the production of pro-inflammatory markers which enter the bloodstream from the gingival submucosa. The result is the same: the development of an immune inflammatory response and/or the local suppression of growth factors in the fetal-placental unit, which in turn triggers labor. ${ }^{14}$ For Sanz et al. ${ }^{10}$ specifically two major pathways have been identified, one direct, in which oral microorganisms and/or their components reach the foetal-placental unit and one indirect, in which inflammatory mediators circulate and impact the foetal-placental unit. $^{10}$

\section{Periodontal disease and pregnancy}

The relationship between periodontal disease and adverse pregnancy outcome remains in debate after all these years. Some authors have suspected that pathogens causing periodontal disease may translocate to the amniotic cavity and so contribute to triggering an adverse pregnancy outcome. ${ }^{15}$ However, Radochova et al. ${ }^{16}$ suggested that, the presence of microbial invasion of the amniotic cavity (MIAC) and intra-amniotic inflammation (IAI) was not related to the periodontal status of women with preterm prelabor rupture of membranes (PPROM). ${ }^{16}$ In the middle of this discrepancy it has been suggested that changes in immunity and/or hormone levels during gestation induce changes in the composition of the microbiota, which leads to increased inflammatory response in mucosal surfaces. Altogether, these data provide evidences that physiological changes associated with pregnancy lead to shifts in the microbial communities colonizing mucosa surfaces, which then induce pro-inflammatory immune responses. Thus, the already dysbiotic microbiota in subgingival sites of PD patients would be submitted to additional factors promoting imbalance during pregnancy, increasing its pathogenic potential to induce gingival inflammation. ${ }^{17}$ In women with periodontitis, the infected periodontal tissues may act as reservoirs of bacteria and their products that can disseminate to the fetus-placenta unit. In severe periodontitis patients, the infection agents and their products are able to activate inflammatory signaling pathways locally and in extra-oral sites, including the placenta-fetal unit, which may not only induce preterm labor but also lead to pre-eclampsia and restrict intrauterine growth. Despite these evidences, the effectiveness of periodontal treatment in preventing gestational complications was still not established since it may be influenced by several factors such as severity of disease, composition of microbial community, treatment strategy, and period of treatment throughout pregnancy. ${ }^{17}$ There has been extensive interest in the Th1:Th2 dichotomy for the maintenance of successful pregnancy. A trend towards the Th2 cytokine profile and a suppression of the Th1 cytokine profile appears to exist both in the peripheral blood but more significantly at the maternal fetal interface. Activation of the proinflammatory Th1 profile rather than suppression of the Th2 profile-is apparent in preterm labour and thus should be considered as the logical target for immunomodulating therapies for the prevention of preterm labour and improving neonatal outcome. ${ }^{18}$ Considering Th1 is a cell mediated immunity and Th2 is a humoral effector mechanism. Certain infectious diseases, even sub-clinical infections, may result in enhanced IL-12 production and in turn an overall shift toward type 1 bias. Th- 1 response leads to the activation of decidual macrophages, which secrete toxic levels of nitric oxide and TNF- $\alpha .{ }^{18}$

\section{Pathogens involved in PD}

As is well known, bacterial invasion in subgingival sites especially of Gram-negative organisms are initiators for periodontal diseases. The periodontal pathogens with persistent inflammation lead to destruction of periodontium. The factors like low socio-economic status, mother's age, race, multiple births, tobacco and drug-abuse may be found to increase risk of adverse pregnancy outcome. However, the same are less correlated with Preterm Low Birth Weight cases (PLBW). Even the invasion of both aerobic and anaerobic may lead to inflammation of gastrointestinal tract and vagina hence contributing to pre-term low-birth weight. The biological mechanisminvolved between PLBW and maternal periodontitis is the translocation of chemical mediators of inflammation. Pre-eclampsia is one of the commonest causes of both maternal and fetal morbidity as it is characterized by hypertension and hyperprotenuria. ${ }^{19}$ Maternal periodontal disease with systemic inflammation as measured by $\mathrm{C}$-reactive protein is associated with an increased risk for preeclampsia. ${ }^{20}$ Machado et al. ${ }^{21}$ provided a longitudinal overview of the subgingival bacterial microbiome using fluorescence in situ hybridization technique, in women in the second trimester of pregnancy (between 14 and 24 weeks), and $48 \mathrm{~h}$ and 8 weeks postpartum. Of 31 women evaluated during pregnancy, 24 returned for the 48-h and 18 for their 8-week exams postpartum. Microbial analysis showed a statistically significant decrease in cell count over the study period for Prevotella nigrescens. $P$. intermedia, Campylobacter rectus, and Porphyromonas gingivalis also decrease, although not significantly, and Aggregatibacter actinomycetemcomitans increased. No significant changes were found for Fusobacterium nucleatum, Treponema denticola, orTannerella forsythia. ${ }^{21}$ Actinomyces infections in pregnancy are rare but, if they occur, have been linked primarily with preterm deliveries. ${ }^{22}$ Blanc et al. ${ }^{23}$ found the placentas from women with periodontitis showed a higher prevalence of periodontopathogens compared to those from women without periodontitis. Samples showed low prevalences of Actinomyces israelii, Parvimonas micra and Tannerella forsythia. An association was found between Eikenella corrodens in placenta and periodontitis. The most ubiquitous bacterium, Fusobacterium nucleatum, was more prevalent in mothers with periodontitis and preterm births and/or low birthweight neonates. Porphyromonas gingivalis, Treponema denticola, Prevotella intermedia and Aggregatibacter actinomycetemcomitans were not detected. ${ }^{23}$

\section{Treatment in pregnancy}

There is no agreement between researchers about treatment of PD in pregnancy. Schwendicke et al. ${ }^{24}$ stated that providing periodontal treatment to pregnant women could potentially reduce the risks of perinatal outcomes, especially in mothers with high risks. Conclusive evidence could not be reached due to risks of bias, risks of random errors, and unclear effects of confounding. Further randomized clinical trials are required for this author. ${ }^{24}$ But for others, some evidence from observational studies suggests that periodontal intervention may reduce adverse pregnancy outcomes. ${ }^{25}$ Although Rosa et al. ${ }^{26}$ add that treatment of periodontal disease with scaling and root planning cannot be considered to be an efficient way of reducing the incidence 
of preterm birth. Women may be advised to have periodical dental examinations during pregnancy to test their dental status and may have treatment for periodontal disease. ${ }^{26}$ However, they should be told that such treatment during pregnancy is unlikely to reduce the risk of preterm birth or low birthweight infants ${ }^{27}$ and primary periodontal care during pregnancy cannot be considered an efficient way of reducing the incidence of preterm birth. ${ }^{26}$ On the other hand, George et al. ${ }^{28}$ said cumulative evidence suggests that periodontal treatment during pregnancy may reduce preterm birth and low birth weight incidence. Nevertheless, these findings need to be further validated through larger more targeted randomized control trials. ${ }^{28}$

\section{Is it effective to give antibiotics for PD in pregnancy?}

Attempts at elimination of infectious agents often do not represent a definitive therapy in periodontitis, requiring the administration of more sophisticated biological treatment modalities. A thorough understanding of the host inflammatory response in periodontal pathogenesis presents the opportunity for exploiting new treatment strategies for periodontitis by means of host response modulation. The rationale behind this approach is to aid the host in its fight against infectious agents by supplementing the natural inherent defense mechanism or to modify its responses by changing the course of inflammatory systems. Therefore, pharmaceutical inhibition of host response pathways that mimic endogenous antiinflammatory mechanisms may prove to be an effective strategy for treating periodontal diseases. This would require the development of polypharmaceutical approaches controlling all pathways associated with inflammation and tissue destruction. ${ }^{4}$ Thinkhamrop et al. ${ }^{29}$ in their research reported antibiotic prophylaxis did not reduce the risk of preterm prelabour rupture of membranes or preterm delivery (apart from in the subgroup of women with a previous preterm birth who had bacterial vaginosis). Antibiotic prophylaxis given during the second or third trimester of pregnancy reduced the risk of postpartum endometritis, term pregnancy with pre-labour rupture of membranes and gonococcal infection when given routinely to all pregnant women. Substantial bias possibly exists in the review's results because of a high rate of loss to follow-up and the small numbers of studies. There is also insufficient evidence on possible harmful effects on the baby. Therefore, there is not enough evidence to support the use of routine antibiotics during pregnancy to prevent infectious adverse effects on pregnancy outcomes. ${ }^{29}$ It is interesting that PD and bacterial vaginosis share microbiological similarities, and both conditions are associated with spontaneous preterm labor and preterm birth. In addition, PD and bacterial vaginosis have been linked through gene polymorphism. Studies evaluating antibiotic administration to eradicate PD and/or bacterial vaginosis responsible organisms, and minimize the risk of preterm births have yielded conflicting results. With respect to bacterial vaginosis, the timing and the choice of antibiotic administration might partly explain the conflicting results. The use of scaling and/or root planning for women with periodontal disease appears to reduce the risk of preterm birth, but routine administration of antibiotics has not demonstrated any impact on preterm birth and the contribution of periodontal disease and bacterial vaginosis are needed. ${ }^{30}$

\section{Dentists and gyneco-obstetrics}

Luc et al. ${ }^{31}$ did a research to analyze knowledge and practices of dentists regarding dental care during pregnancy using a questionnaire.
Their results showed $53.9 \%$ of pregnant women are not referred by an obstetrician. Only $59.8 \%$ of practitioners believe that dental anaesthesia is not contraindicated, and most practitioners believe that the best time for care is the second trimester or after pregnancy. During pregnancy, practitioners realize the motivation for hygiene $(90.7 \%)$, drainage of an abscess (82.2\%), scaling (76.1\%), the placement of a splint $(74.1 \%)$, the treatment of caries $(70 \%)$, and the removable prosthesis $(67.2 \%)$. These results indicate the existence of a gap between the care of pregnant women and the state of the art in dental science, despite current clinical recommendations to deliver all necessary care to pregnant patients during the first, second and third trimester. Moreover, the results highlight the need to reinforce the initial training of dental students, but also to develop the training for practitioners in this field. ${ }^{31}$ In a study rolled in Murcia, Spain, the authors found the vast majority of dentists $(81.3 \%)$ agreed that oral care should be part of general healthcare for pregnant women. However, discrepancies were found in knowledge and attitudes to practice..$^{32}$ Also Vieira et al. ${ }^{33}$ reported dentists have doubts and fears about the care of pregnant women, to a greater or lesser degree, especially with regard to the use of X-rays, prescriptions and ideal gestational period of treatment. ${ }^{33}$ Pregnancy is a time of particular vulnerability in terms of oral health due to complex physical and physiological changes ${ }^{34}$ nevertheless by itself is not a reason to defer routine dental care and necessary treatment for oral health problems. Diagnosis and treatment, including needed dental X-rays, can be undertaken safely during the first trimester of pregnancy. Needed treatment can be provided throughout the remainder of the pregnancy; however, the time period between the 14th and 20th week is considered ideal. ${ }^{35}$ General practitioners and midwives are poorly informed about the impact of poor maternal oral health and rarely initiate this topic during prenatal care. Obstetricians/gynecologists are well informed about perinatal oral health and are supportive of dental procedures, but because of lack of training in this area and competing health demands they seldom focus on oral health care during their prenatal care. In consequence, there is no real consensus exists among dentists and prenatal care practitioners with respect to oral health care during pregnancy. This issue poses a significant deterrent for pregnant women seeking dental care. Practice guidelines in perinatal oral health are needed for health professionals to emphasize this important aspect of prenatal care. ${ }^{36}$ Physicians and dentists are responsible for providing professional medical care and treatment following clinical guidelines especially for pregnancy. It is also an opportune time in pregnancy to educate women about oral health care and preventing oral problems in young children. ${ }^{34}$ It is known that the periodontal health and oral health related quality of life of pregnant women is poorer than nonpregnant women. ${ }^{37}$

\section{Importance of guidelines in this topic}

In the same topic, a research carried out in Poland showed low maternal awareness regarding health-related behaviors, which presents a serious challenge to public health care. Only $46.7 \%$ of women informed their dentist about their pregnancy, and these were more often older participants. ${ }^{11}$ Antenatal care providers are now recommended to promote oral health during pregnancy and provide dental referrals. However, midwives in Australia are not trained to undertake this role. To address this shortcoming, an online evidence based midwifery initiated oral health education program was systematically developed as a professional activity. ${ }^{38}$ Dilemmatic attitude and practice behavior of dentists should be streamlined to render right care to the pregnant women at the correct time. 
Perplexing perception toward oral health care by pregnant women must be overcome to orient them to understand the impact of oral health on their general systemic health. ${ }^{39}$ Oral disease prevention strategies should be incorporated in chronic systemic disease preventive initiatives to curtail the burden of disease in populations. The reduction in the incidence and prevalence of periodontal disease can reduce its associated systemic diseases and can also minimize their financial impact on the health-care systems. ${ }^{2}$ Pregnancy had an influence only on the gingiva and not on periodontal attachment levels. However, oral health status and perceived oral health related quality of life were poorer among pregnant women. There is a real need for highlighting the importance of conserving oral health during pregnancy. ${ }^{40}$ In this min-review we have found that despite of the independent increase of knowledge in both the periodontal disease and the perinatal disease, there is still no clear consensus about through which physiopathological pathway this diseases are linked, moreover how the management of the first would affect the second one. What is clear is that periodontitis and low access to dental care might also share some environmental risk factor in common with preterm delivery, low birth weight and preeclampsia, leaving us to consider the cofounding theory as an important one to be considered. We recognize that health care must be contemplated as a whole, and dental care should be an essential part of prenatal health promoting measures.

\section{Funding}

None.

\section{Acknowledgments}

None.

\section{Conflicts of interest}

Author declares that there is no conflict of interest.

\section{References}

1. Martelli ML, Brandi ML, Martelli M, et al. Periodontal disease and women's health. Curr Med Res Opin. 2017;33(6):1005-1015.

2. Nazir MA. Prevalence of periodontal disease, its association with systemic diseases and prevention. Int $J$ Health Sci (Qassim). 2017;11(2):72-80.

3. Cochran D. Periodontology in the age of inflammation: a changing landscape. J Am Coll Dent. 2009;76(1):23-26.

4. Deo V, Bhongade ML. Pathogenesis of periodontitis: role of cytokines in host response. Dent Today. 2010;29(9):60-69.

5. Malinova M. Periodontal disease and preterm birth. Akush Ginekol (Sofiia). 2013;52 Suppl:43-48.

6. Russell SL, Mayberry LJ. Pregnancy and oral health: a review and recommendations to reduce gaps in practice and research. MCN Am J Matern Child Nurs. 2008;33(1):32-37.

7. Mattheus D, Shannon M, Lim E, et al. The Association Between Socio-demographic Factors, Dental Problems, and Preterm Labor for Pregnant Women Residing in Hawai'i. Hawaii J Med Public Health. 2016;75(8):219-227.

8. Varadan M, Ramamurthy J. Association of periodontal disease and pre-term low birth weight infants. $J$ Obstet Gynaecol India. 2015;65(3):167-171.

9. Riggs E, Yelland J, Shankumar R, et al. We are all scared for the baby': promoting access to dental services for refugee background women during pregnancy. BMC Pregnancy and Childbirth. 2016;16:12.
10. Sanz M, Kornman K. Periodontitis and adverse pregnancy outcomes: consensus report of the Joint EFP/AAP Workshop on Periodontitis and Systemic Diseases. J Periodontol. 2013;84(4 Supp1):S164-S169.

11. Górniaczyk A, Czech-Szczapa B, Sobkowski M, et al. Maternal healthrelated behaviours during pregnancy: a critical public health issue. Eur $J$ Contracept Reprod Health Care. 2017;22(4):321-325.

12. Chen S, Zhu R, Zhu H, et al. The prevalence and risk factors of preterm small-for-gestational-age infants: a population-based retrospective cohort study in rural Chinese population. BMC Pregnancy Childbirth. 2017; 17(1):237.

13. Liu L, Johnson HL, Cousens S, et al. Global, regional, and national causes of child mortality: an updated systematic analysis for 2010 with time trends since 2000. Lancet. 2012;379(9832):2151-2161.

14. Puertas A, Magan-Fernandez A, Blanc V, et al. Association of periodontitis with preterm birth and low birth weight: a comprehensive review. $J$ Matern Fetal Neonatal Med. 2017;28:1-6.

15. Andonova I, Iliev V, Zivkovic N, et al. Can oral anaerobic bacteria cause adverse pregnancy outcomes? Pril (Makedon Akad Nauk Umet Odd Med Nauki). 2015;36(1):137-143.

16. Radochova V, Kacerovska Musilova I, Stepan M, et al. Periodontal disease and intra-amniotic complications in women with preterm prelabor rupture of membranes. J Matern Fetal Neonatal Med. 2017;4:1-10.

17. Zi MY, Longo PL, Bueno-Silva B, et al. Mechanisms involved in the association between periodontitis and complications in pregnancy. Front Public Health. 2015;2:290.

18. Sykes L, MacIntyre DA, Yap XJ, et al. The Th1:Th2 dichotomy of Pregnancy and Preterm labour. Mediators of Inflammation. 2012;967629:1-12.

19. Parihar AS, Katoch V, Rajguru SA, et al. Periodontal Disease: A Possible Risk-Factor for Adverse Pregnancy Outcome. J Int Oral Health. 2015;7(7):137-142.

20. Ruma M, Boggess K, Moss K, et al. Maternal periodontal disease, systemic inflammation, and risk for preeclampsia. Am J Obstet and Gynecol. 2008;198(4):389.e1-389.e5.

21. Machado FC, Cesar DE, Apolônio AC, et al. Longitudinal study on clinical and microbial analysis of periodontal status in pregnancy. Braz Oral Res. 2016;30(1):e87.

22. Estrada SM, Magann EF, Napolitano PG. Actinomyces in Pregnancy: A Review of the Literature. Obstet Gynecol Surv. 2017;72(4):242-247.

23. Blanc V, O’Valle F, Pozo E, et al. Oral bacteria in placental tissues: increased molecular detection in pregnant periodontitis patients. Oral Dis. 2015;21(7):905-912.

24. Schwendicke F, Karimbux N, Allareddy V, et al. Periodontal treatment for preventing adverse pregnancy outcomes: a meta- and trial sequential analysis. PLoS ONE. 2015;10(6):e0129060.

25. Iheozor-Ejiofor Z, Middleton P, Esposito M, et al. Treating periodontal disease for preventing adverse birth outcomes in pregnant women. Cochrane Database Syst Rev. 2017;6:CD005297.

26. Rosa MI, Pires PD, Medeiros LR, et al. Periodontal disease treatment and risk of preterm birth: a systematic review and meta-analysis. Cad Saude Publica. 2012;28(10):1823-1833.

27. Polyzos NP, Polyzos IP, Zavos A, et al. Obstetric outcomes after treatment of periodontal disease during pregnancy: systematic reviewand metaanalysis. BMJ. 2010;341:c7017.

28. George A, Shamim S, Johnson M, et al. Periodontal treatment during pregnancy and birth outcomes: a meta-analysis of randomised trials. Int $J$ Evid Based Healthc. 2011;9(2):122-147. 
29. Thinkhamrop J, Hofmeyr GJ, Adetoro O, et al. Antibiotic prophylaxis during the second and third trimester to reduce adverse pregnancy outcomes and morbidity. Cochrane Database Syst Rev. 2015;1:CD002250.

30. Sanu O, Lamont RF. Periodontal disease and bacterial vaginosis as genetic and environmental markers for the risk of spontaneous preterm labor and preterm birth. J Matern Fetal Neonatal Med. 2011;24(12):1476-1485.

31. Luc E, Coulibaly N, Demoersman J, et al. Dental care during pregnancy. Schweiz Monatsschr Zahnmed. 2012;122(11):1047-1063.

32. López-Jornet P, Camacho-Alonso F, Sanchez-Siles M, et al. Oral and dental health in pregnant women: attitudes among dentists in southeastern Spain. N Y State Dent J. 2014;80(1):38-41.

33. Vieira DR, de Oliveira AE, Lopes FF, et al. Dentists' knowledge of oral health during pregnancy: a review of the last 10 years' publications. Community Dent Health. 2015;32(2):77-82.

34. Liwei Z, Jing Z, Yong Y, et al. Management of oral diseases during pregnancy. Hua Xi Kou Qiang Yi Xue Za Zhi. 2017;35(2):113-118.

35. Kumar J, Samelson R. Oral health care during pregnancy recommendations for oral health professionals. N Y State Dent J. 2009;75(6):29-33.
36. George A, Shamim S, Johnson M, et al. How do dental and prenatal care practitioners perceive dental care during pregnancy? Current evidence and implications. Birth. 2012;39(3):238-247.

37. Geevarghese A, Baskaradoss JK, Sarma PS. Oral Health-Related Quality of Life and Periodontal Status of Pregnant Women. Matern Child Health J. 2017;21(8):1634-1642.

38. George A, Lang G, Johnson M, et al. The evaluation of an oral health education program for midwifes in Australia. Women Birth. 2016;29(3):208-213.

39. Jeelani S, Khader KA, Rangdhol RV, Dany A, Paulose S (2015) Coalition of attitude an practice behaviors among dental practitioners regarding pregnant patient's oral health and pregnant patient's perception toward oral health in and around Pondicherry. J Pharm Bioallied Sci 7(Suppl 2): S509-S512.

40. Shah AF, Batra M, Qureshi A (2017) Evaluation of Impact of Pregnancy on Oral Health Status and Oral Health Related Quality of Life among Women of Kashmir Valley. J Clin Diagn Res 11(5): ZC01-ZC04. 